Taxonomía y sistemática

\title{
Licopodios y helechos de San Juan Colorado, Oaxaca, México
}

\author{
Lycopods and ferns of San Juan Colorado, Oaxaca, Mexico \\ Aniceto Mendoza-Ruiz*, Jacqueline Ceja-Romero y María Isabel Mejía-Marín
}

Área de Botánica Estructural y Sistemática Vegetal, Departamento de Biología, Universidad Autónoma Metropolitana, Unidad Iztapalapa, Av. San Rafael Atlixco Núm. 186, Colonia Vicentina, Iztapalapa, 09340 Ciudad de México, México

Recibido el 8 de febrero de 2016; aceptado el 28 de septiembre de 2016

Disponible en Internet el 23 de febrero de 2017

\section{Resumen}

Con el objetivo de elaborar el listado florístico de los licopodios y helechos que habitan en el municipio de San Juan Colorado, área poco explorada y localizada en el distrito de Jamiltepec, Oaxaca, se realizaron diversos viajes de recolección durante los años 2012 a 2014 . Como resultado del trabajo se registraron 15 familias, 33 géneros y 68 especies para el municipio y se incrementó a 81 el número de especies presentes en el distrito, lo que representa el $11.8 \%$ del total reportado a nivel estatal para este grupo de plantas. Los tipos de vegetación con mayor riqueza específica fueron el bosque tropical subcaducifolio y el bosque mesófilo de montaña. Se registraron por primera vez para Oaxaca: Adiantum decoratum, Anemia intermedia y $\times$ Hemionanthes gryphus. El $79 \%$ de los taxones son terrestres. La comparación de estos resultados con la información previa disponible a nivel distrital y municipal pone de manifiesto la importancia de los estudios florísticos a nivel local para avanzar en el conocimiento de la diversidad vegetal de Oaxaca.

(C) 2017 Universidad Nacional Autónoma de México, Instituto de Biología. Este es un artículo Open Access bajo la licencia CC BY-NC-ND (http://creativecommons.org/licenses/by-nc-nd/4.0/).

Palabras clave: Diversidad; Distribución; Nuevos registros; Pteridofitas; Riqueza específica

\begin{abstract}
In order to carry out a checklist of the lycopods and ferns from the municipality of San Juan Colorado, an unexplored area located in the District of Jamiltepec, Oaxaca, several field trips were made from 2012 to 2014. A list of 15 families, 33 genera, and 68 species was recorded for the municipality, which increased to 81 the taxa in the District and represents $11.8 \%$ of the total reported at the state level for this group of plants. The vegetation types with higher species richness were the semi-deciduous tropical forest and the cloud forest. We registered for the first time in Oaxaca: Adiantum decoratum, Anemia intermedia, and $\times$ Hemionanthes gryphus. Seventy nine per cent of the taxa are terrestrial. Comparison of these results with previous information available at a district and a municipal level shows the importance of local floristic studies to advance the knowledge of plant diversity of Oaxaca.
\end{abstract}

(C) 2017 Universidad Nacional Autónoma de México, Instituto de Biología. This is an open access article under the CC BY-NC-ND license (http://creativecommons.org/licenses/by-nc-nd/4.0/).

Keywords: Diversity; Distribution; New records; Pteridophytes; Specific richness

\section{Introducción}

Oaxaca es uno de los estados con mayor riqueza de plantas vasculares en el país, con 8,903 especies (García-Mendoza y

\footnotetext{
* Autor para correspondencia.

Correo electrónico: amr@xanum.uam.mx (A. Mendoza-Ruiz).

La revisión por pares es responsabilidad de la Universidad Nacional Autónoma de México.
}

Meave, 2011); sin embargo, esta cifra dista de ser definitiva, ya que a pesar del intenso trabajo florístico que se ha realizado en la entidad, al menos el $50 \%$ de su superficie todavía no ha sido inventariada, por lo que es necesario impulsar el trabajo de recolección en las regiones faltantes (García-Mendoza, 2004).

En lo que a licopodios y helechos se refiere, se cuenta con el tratamiento taxonómico a nivel estatal realizado por Mickel y Beitel (1988) y con los fascículos que como parte del proyecto Flora del Valle de Tehuacán-Cuicatlán se han publicado para 
diversas familias (Riba y Lira, 1996; Velázquez-Montes, 2009, 2010). Existe también información a partir de listados florísticos distritales y municipales que incluyen a las plantas vasculares sin semilla (Acosta, Flores, Saynes, Aguilar y Manzanero, 2003; Aparicio-Lechuga y Vargas-Fuerte, 2010; Carrillo-Hernández, 2013; Figueroa-Brito y Guzmán-Rivera, 2005; Guízar-Nolazco, 2011; Pérez-García, Meave y Gallardo, 2001; Reyes-Santiago, 1993; Santiago-Cruz, 2013; Solano-Hernández, 1997; TorresColín, Torres-Colín, Dávila-Aranda y Villaseñor-Ríos, 1997) y, sobre todo, se cuenta con los trabajos de síntesis y análisis que realizaron Tejero-Díez y Mickel (2004, 2011a, 2011b), en los que contabilizaron 683 especies para el estado. Sin embargo, resulta evidente que, de manera similar a lo que ocurre con los demás grupos de plantas, el trabajo de exploración modifica invariablemente los datos sobre la riqueza y distribución de los licopodios y helechos registrados para Oaxaca.

San Juan Colorado es uno de los 24 municipios que conforman el distrito de Jamiltepec, zona para la cual García-Mendoza (2004) y Salas-Morales, Schibli, Nava-Zafra y Saynes-Vásquez (2007) indican la necesidad urgente de realizar trabajo de exploración, debido a que no cuenta con estudios de vegetación o florísticos de áreas específicas. En particular para los licopodios y helechos, los datos generados sobre el número de especies que habitan la zona se restringen a las 27 especies registradas por Tejero-Díez y Mickel (2004) para todo el distrito. La falta de información sobre el grupo en la zona motivó la realización del presente estudio, cuyo objetivo fue conocer la riqueza, composición florística, distribución por tipo de vegetación y hábito de los licopodios y helechos que crecen en el municipio de San Juan Colorado, complementando así la información que se tiene sobre este grupo de plantas a nivel distrital y estatal.

\section{Materiales y métodos}

El municipio de San Juan Colorado forma parte del distrito de Jamiltepec, en la región costa del estado de Oaxaca. Se localiza entre los $16^{\circ} 25^{\prime}$ y $16^{\circ} 35^{\prime} \mathrm{N}, 97^{\circ} 51^{\prime}$ y $98^{\circ} 00^{\prime}$ O (Inegi, 2010). Ocupa una superficie aproximada de $86 \mathrm{~km}^{2}$, lo que representa el $0.13 \%$ de la superficie total del estado. Limita al norte con el municipio de La Reforma, al este con Santiago Ixtayutla y San Agustín Chayuco, al sur con San Lorenzo y Pinotepa de Don Luis y al oeste con San Pedro Atoyac y San Pedro Jicayán. Cuenta con las agencias municipales de Santa María Nutío, Nuevo Progreso, Peñas Negras, San Pedro Siniyuvi, la agencia de policía de El Terrero y el núcleo rural de Agua Fría (fig. 1) (Inegi, 2010; Consejo de Desarrollo Social Municipal de San Juan Colorado, 2011).

San Juan Colorado forma parte de la provincia fisiográfica Sierra Madre del Sur, subprovincia Costas del Sur. El clima predominante es el cálido subhúmedo con lluvias en verano. La temperatura promedio anual oscila entre 22 y $28^{\circ} \mathrm{C}$ y la precipitación acumulada anual, entre 1,500 y 2,500 $\mathrm{mm}$. El intervalo altitudinal va de 200 a 1,400 m snm, con 3 principales elevaciones: Yucu-savi (cerro de La Lluvia), Yucu-cha'a (cerro de El Bule) y Yucu tutú (cerro de La Esquina) (Inegi, 2005, 2010; Consejo de Desarrollo Social Municipal de San Juan Colorado, 2011).
Según el Inegi (2010), el 38.96\% de la superficie del municipio está ocupada por pastizal cultivado, el $26.60 \%$ por bosques, el $26.14 \%$ por selvas y el resto corresponde a las zonas agrícola y urbana. Mejía-Marín (2014) reporta que de acuerdo con Rzedowski (2006) los tipos de vegetación presentes en el área son: bosque de Quercus, bosque mixto de Pinus-Quercus, bosque de galería, bosque mesófilo de montaña, bosque tropical caducifolio, bosque tropical subcaducifolio, matorral, pastizal y vegetación acuática.

Con el propósito de obtener material botánico de licopodios y helechos, durante los años 2012 a 2014 se realizaron 6 viajes de recolección a localidades de las distintas agencias municipales de San Juan Colorado. Los ejemplares, una vez determinados, se depositaron en el Herbario UAMIZ. Con la finalidad de complementar el listado, se revisaron los herbarios ENCB, MEXU, OAX, SERO y UAMIZ (Thiers, continuamente actualizado) y se consultaron las bases de datos del University of Arizona Herbarium (ASU, http://nhc.asu.edu/vpherbarium/), Digital specimen images of Herbarium Berolinense (Röpert, 2000), The New York Botanical Garden (NY, http://sciweb.nybg. org/science2/hcol/allvasc/index.asp.html) y Tropicos.org del Missouri Botanical Garden (http://www.tropicos.org/). Con la información obtenida se elaboró el catálogo de las especies de licopodios y helechos que crecen en el municipio, enlistándolas en orden alfabético por familia, género y especie de acuerdo con el sistema de clasificación propuesto por Smith et al. (2006). Los nombres de los taxones se corrigieron con literatura actualizada (p. ej., Øllgaard, 2012; Smith y Tejero-Díez, 2014) y se cotejaron con The Plant List (2013) y The International Plant Names Index (2015). En cada caso, se indicó el hábito considerando las siguientes categorías: terrestres, rupícolas, epífitas o acuáticas.

Para comparar los resultados obtenidos con los registrados para otras regiones de Oaxaca, se estimó el número de especies por $\mathrm{km}^{2}$ (riqueza relativa).

\section{Resultados}

A partir de la determinación de los 158 ejemplares recolectados en las distintas agencias municipales de San Juan Colorado, se obtuvo una lista de 2 familias, 2 géneros y 5 especies de licopodios y 13 familias, 32 géneros y 63 especies de helechos (apéndice), lo que representa el 9.9\% del total de especies registradas hasta el momento a nivel estatal. El intervalo altitudinal en el que se recolectaron las plantas va de 250 a $950 \mathrm{~m}$, con el mayor número de taxones presentes entre los 850 y los $950 \mathrm{~m}$. Las familias con más especies son Pteridaceae con 20 y Thelypteridaceae con 9. Los géneros con mayor número de especies son Adiantum, Thelypteris y Anemia con 9, 8 y 6, respectivamente. La riqueza fue de 0.79 especies por $\mathrm{km}^{2}$ (tabla 1 ).

El bosque tropical subcaducifolio es el tipo de vegetación en el que habitan más especies (40), le siguen el bosque mesófilo de montaña (25), de Quercus (21) y mixto de Pinus-Quercus (16). La vegetación secundaria (10), el bosque tropical caducifolio (3) y el bosque de galería (1) son las asociaciones vegetales en las que habitan menor número de especies. 


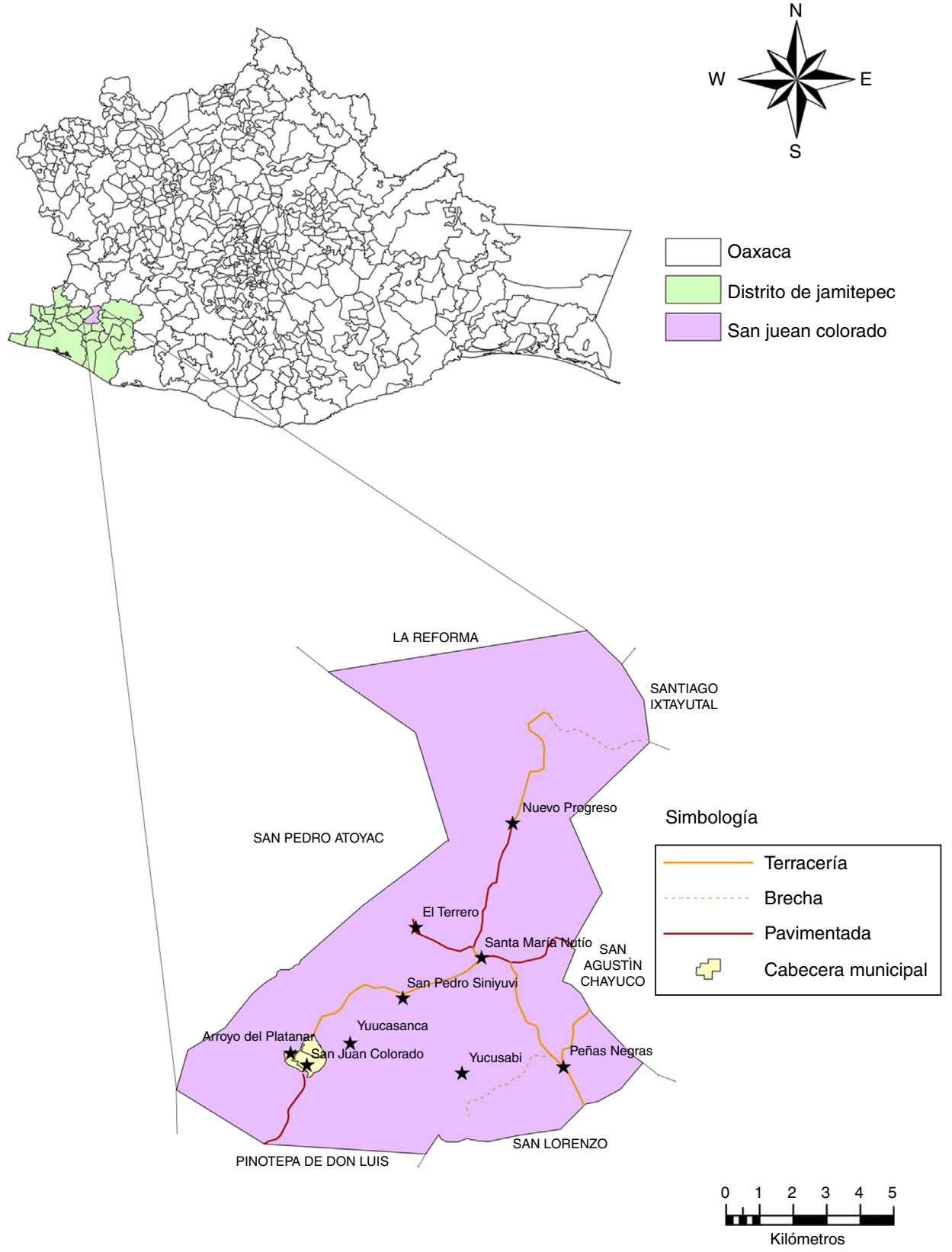

Figura 1. Mapa del municipio de San Juan Colorado, área de estudio.

La agencia municipal con más riqueza específica es Nuevo Progreso con 42 taxones, seguida de Agua Fría, Peñas Negras y San Juan Colorado con 24 cada una y, por último, Santa María Nutío con 3.

De las 68 especies recolectadas, 54 son terrestres, 4 rupícolas, 2 rupícolas o terrestres, 4 epífitas, 3 epífitas o rupícolas y solo 1 es subacuática. Por otra parte, son nuevos registros para el estado Adiantum decoratum Maxon \& Weath, Anemia intermedia Copel ex M.E. Jones y $\times$ Hemionanthes gryphus Mickel (Mickel). Las especies Cyathea costaricensis (Mett. ex Kuhn) Domin y Sphaeropteris horrida (Liebm.) R.M. Tryon se encuentran en la NOM-ECOL-059 (Semarnat, 2010), la primera bajo la categoría de en peligro de extinción y la segunda como sujeta a protección especial, debido a que las poblaciones están decreciendo por la tala inmoderada de los bosques. En particular, en San Juan Colorado estos taxones son escasos y solo crecen en pequeños manchones de bosques conservados.

\section{Discusión}

Diversos factores geográficos y sociales han originado que, para 11 de los 30 distritos en que se divide Oaxaca, la información florística sea escasa o inexistente (García-Mendoza, 2004). $\mathrm{Si}$ consideramos la magnitud del esfuerzo que se requiere para cubrir adecuadamente el trabajo de exploración botánico en algunas regiones del estado, cobran particular importancia los estudios que se realizan a nivel local. Prueba de lo anterior es que de las 68 especies de licopodios y helechos que se reportan 
Tabla 1

Comparación de la riqueza de especies de licopodios y helechos del municipio de San Juan Colorado con otras zonas del estado de Oaxaca.

\begin{tabular}{|c|c|c|c|c|}
\hline Área de estudio & Fuente & Extensión & Núm. spp. & $\begin{array}{l}\text { Riqueza relativa } \\
\text { (número } \mathrm{spp} / \mathrm{km}^{2} \text { ) }\end{array}$ \\
\hline Municipio San Juan Colorado & Este trabajo & $86 \mathrm{~km}^{2}$ & 68 & 0.79 \\
\hline Municipio San Juan Mixtepec & Reyes-Santiago, 1993 & 3,500 ha & 25 & 0.71 \\
\hline Municipio Asunción Cuyotepeji & Solano-Hernández, 1997 & $91 \mathrm{~km}^{2}$ & 19 & 0.21 \\
\hline Cerro Guiengola & Torres-Colín et al., 1997 & 4,530 ha & 3 & 0.07 \\
\hline Región de Nizanda & Pérez-García et al., 2001 & $85 \mathrm{~km}^{2}$ & 24 & 0.28 \\
\hline Zona semiárida de la cuenca alta del río Tehuantepec & Acosta et al., 2003 & sin precisar & 5 & - \\
\hline Región de Zimatán & Salas-Morales et al., 2003 & 71,339 ha & 49 & 0.07 \\
\hline Capulalpam de Méndez & Figueroa-Brito y Guzmán-Rivera, 2005 & 3,850 ha & 97 & 2.52 \\
\hline Bosques mesófilos de la sierra Mazateca de Oaxaca & Lorea-Hernández y Munn-Estrada, 2005 & $1,050 \mathrm{~km}^{2}$ & 80 & 7.62 \\
\hline Parque Nacional Huatulco & Salas-Morales et al., 2007 & $6,374.98$ ha & 4 & 0.06 \\
\hline Municipio Ciudad Ixtepec & Aparicio-Lechuga y Vargas-Fuerte, 2010 & $294.6 \mathrm{~km}^{2}$ & 8 & 0.03 \\
\hline Porción noroeste del distrito Mixe & Carrillo-Hernández, 2013 & sin precisar & 33 & - \\
\hline Comunidad de Santa Cruz Yagavila & Santiago-Cruz, 2013 & sin precisar & 88 & - \\
\hline
\end{tabular}

en este trabajo, solo 14 habían sido registradas con anterioridad para el distrito de Jamiltepec por Tejero-Díez y Mickel (2011a, 2011b), por lo que las 54 restantes son nuevos registros (apéndice). Con este aporte, el número de taxones a nivel distrital aumenta a 81 , lo que equivale al $11.8 \%$ de la riqueza total del estado, cifra similar a la observada por Tejero-Díez y Mickel (2004) en los distritos de Sola de Vega (81) y Putla (83).

A nivel municipal, todos los ejemplares de licopodios y helechos obtenidos en este trabajo constituyen primeros registros, ya que, durante la revisión de las distintas colecciones de herbario y bases de datos, no se localizó material del grupo en cuestión procedente de la zona de estudio. La comparación de los resultados con otros estudios florísticos municipales, que incluyen información sobre pteridofitas (tabla 1), hace evidente la contribución de San Juan Colorado a la diversidad biológica de Oaxaca, ya que no obstante contar con solo $86 \mathrm{~km}^{2}$ de superficie, alberga el $9.9 \%$ de especies que se tienen reportadas para la entidad.

En lo que se refiere a tipo de vegetación, es importante destacar que a pesar de que casi el $50 \%$ de la superficie municipal se encuentra ocupada por pastizales o campos de cultivo (Inegi, 2010), todavía existen algunas áreas de bosques conservados o poco alterados. Esto generalmente es debido a las características topográficas del terreno donde se desarrollan cañadas con pendientes pronunciadas y de difícil acceso, las cuales contribuyen de manera significativa a la conservación de las especies, por lo que es necesario concientizar a la población sobre la importancia de preservarlas.

La riqueza de especies por $\mathrm{km}^{2}$ de San Juan Colorado (tabla 1) es mayor que la obtenida para la región de Zimatán, la cual abarca los municipios de San Miguel de Puerto y San Pedro Huamelula (Salas-Morales, Saynes-Vásquez y Schibli, 2003) o la del Parque Nacional Huatulco, que pertenece al municipio de Santa María Huatulco (Salas-Morales et al., 2007), pero inferior a la registrada en Capulalpam de Méndez y la sierra Mazateca de Oaxaca (Figueroa-Brito y GuzmánRivera, 2005; Lorea-Hernández y Munn-Estrada, 2005). Lo anterior se puede relacionar con el hecho de que en el primer caso la vegetación dominante es el bosque tropical caducifolio, mientras que en el segundo es el bosque mesófilo de montaña, tipo de hábitat en el que Rzedowski (2006) y Smith y TejeroDíez (2014) señalan son más abundantes y diversificados los licopodios y helechos.

En México, la relación entre la riqueza específica de helechos y el gradiente altitudinal se ha documentado para algunas zonas de Veracruz, como los Tuxtlas (Acebey, Krömer, Vázquez-Torres y Tejero-Díez, 2015) y el Cofre de Perote (Carvajal-Hernández, Krömer y Vázquez-Torres, 2015), donde se registran los valores más altos en elevaciones medias entre 750-1,200 $\mathrm{m}$ en el primer caso y entre 1,500-2,500 $\mathrm{m}$ en el segundo, y decrecen hacia los extremos. En San Juan Colorado el mayor número de especies se registró entre los 850-950 m snm, elevación que es el límite superior de la zona de estudio y que coincide con el desarrollo de pequeños manchones de bosque mesófilo de montaña.

En lo que concierne a los nuevos registros para el estado, $A$. decoratum había sido reportado previamente para Quintana Roo, Tabasco, Veracruz y Chiapas, estados colindantes entre sí, por lo que con este trabajo se amplía el área de distribución conocida para la especie. Por otra parte, $\times H$. gryphus se conoce del estado de México, Nayarit, Colima y Michoacán, sin embargo, la falta de registros para Guerrero interrumpe lo que sería una distribución continua a lo largo de la costa del Pacífico, por lo que consideramos que es muy posible que también esté presente en la entidad mencionada. De particular interés resulta $A$. intermedia, especie que solo se conocía de Nayarit y para la que Mickel y Smith (2004) señalan que puede tratarse de un híbrido entre Anemia brandegeea Davenp., reportada de Sinaloa y Anemia clinata Mickel, que se distribuye desde Panamá hasta Bolivia. En el área de estudio no observamos la presencia de ninguna de las 2 especies mencionadas como posibles progenitores, por lo que es necesario contar con más información para explicar la distribución disyunta de la especie.

En el área de estudio la mayoría de las especies son terrestres, lo que concuerda con lo señalado por Acebey et al. (2015), en el sentido de que la riqueza de especies terrestres es mayor en las selvas tropicales de las zonas bajas, mientras que las epífitas son más numerosas en el bosque mesófilo de montaña.

Finalmente, resulta evidente que la realización de estudios florísticos a nivel local permite conocer mejor la riqueza de 
especies y avanzar de manera paulatina en el conocimiento de la diversidad vegetal de Oaxaca.

\section{Agradecimientos}

Damos las gracias a las autoridades de San Juan Colorado y de las distintas agencias municipales por las facilidades prestadas durante la realización del estudio, así como a Evencio Mejía Marín, Adolfo Espejo y Ana Rosa López Ferrari por su invaluable apoyo durante el trabajo de campo. Agradecemos también los valiosos comentarios y sugerencias al manuscrito realizados por Ana Rosa, a los 2 revisores anónimos y a la editora asociada Alejandra Vasco.

\section{Apéndice. Licopodios y helechos del municipio de San Juan Colorado, Oaxaca $(*=$ nuevos registros para el distrito; $* *=$ nuevos registros para el estado; + = NOM-ECOL-059-SEMARNAT-2010; ${ }^{\mathrm{e}}=$ epífito; $^{\text {N }}$ $\mathrm{r}=$ rupícola; $^{\mathrm{s}}=$ subacuático $^{\mathrm{t}}{ }^{\mathrm{a}}=$ terrestre $^{\mathrm{ta}}=$ terrestre arborescente).}

Lycopodiophyta

Lycopodiaceae

* Palhinhaea cernua (L.) Vasc. \& Franco

(Lycopodiella cernua (L.) Pic. Serm.)

Nuevo Progreso: A. Mendoza R. et al., 2125 (UAMIZ); Santa María Nutio: I. Mejía M. et al., 105 (UAMIZ), A. Mendoza R. et al., 2120 (UAMIZ).

Selaginellaceae

*r Selaginella hoffmannii Hieron.

Agua Fría: A. Mendoza R. et al., 2045 (UAMIZ); Nuevo Progreso:

A. Mendoza R. et al., 2148 (UAMIZ).

${ }^{\mathrm{t}}$ Selaginella pallescens (C. Presl) Spring

Peñas Negras: A. Mendoza R. et al., 2022 (UAMIZ).

${ }^{\mathrm{t}}$ Selaginella prolifera Valdespino

Peñas Negras: A. Mendoza R. et al., 2010 (UAMIZ).

*tSelaginella tarda Mickel \& Beitel

SAN Juan Colorado: I. Mejía M. et al., 711 (UAMIZ).

Polypodiophyta

Anemiaceae

${ }^{\mathrm{t}}$ Anemia hirsuta (L.) Sw.

San Juan Colorado: A. Mendoza R. et al., 2059 (UAMIZ).

**t Anemia intermedia Copel.

Nuevo Progreso: A. Mendoza R. et al., 2116 (UAMIZ).

*t Anemia karwinskyana (C. Presl) Prantl.

Nuevo Progreso: A. Mendoza R. et al., 2143 (UAMIZ).

*t Anemia oblongifolia (Cav.) Sw.

Agua Fría: I. Mejía M. et al., 139 (UAMIZ); Nuevo Progreso: A. Mendoza R. et al., 2124 (UAMIZ); Peñas Negras: A. Mendoza R. et al., 2007 (UAMIZ), 2019 (UAMIZ); SAn JuAn Colorado: A.

Mendoza R. et al., 2032 (UAMIZ).

tAnemia pastinacaria Moritz ex Prantl

Agua Fría: I. Mejía M. et al., 125 (UAMIZ); A. Mendoza R. et al., 2036

(UAMIZ); SAN JUAN Colorado: A. Mendoza R. et al., 2034 (UAMIZ)

*t Anemia semihirsuta Mickel

SAN JuAn Colorado:I. Mejía M. et al., 154 (UAMIZ).

Aspleniaceae

* Asplenium formosum Willd.

Agua Fría: A. Mendoza R. et al., 2057 (UAMIZ)
Athyriaceae

*t Athyrium skinneri (Baker) Diels.

Peñas Negras: A. Mendoza R. et al., 2015 (UAMIZ), 2025 (UAMIZ).

${ }^{* \mathrm{r}}$ Diplazium lonchophyllum Kunze

Peñas Negras: A. Mendoza R. et al., 1887 (UAMIZ).

Blechnaceae

*r,t Blechnum gracile Kaulf.

AGUA FRÍA: A. Mendoza R. et al., 2056 (UAMIZ); Nuevo Progreso:

A. Mendoza R. et al., 1866 (UAMIZ), 2108 BIS (UAMIZ); San Juan

Colorado: A. Mendoza R. et al., 1857 (UAMIZ).

* Blechnum occidentale $\mathrm{L}$.

Agua Fría: I. Mejía M. et al., 126 (UAMIZ); A. Mendoza R. et al., 2046 (UAMIZ), 2058 (UAMIZ); Nuevo Progreso: A. Mendoza R. et al.1880 (UAMIZ), 2108 (UAMIZ), 2138 (UAMIZ); SANTA MARÍA Nutio: I. Mejía M. et al., 82 (UAMIZ).

*t Blechnum polypodioides Raddi

Agua fría: A. Mendoza R. et al., 2040 (UAMIZ); Nuevo Progreso: A. Mendoza R. et al., 2126 (UAMIZ); Peñas Negras: A. Mendoza R. et al., 2020 (UAMIZ).

*t Blechnum schiedeanum (Schltdl. ex C. Presl) Hieron.

Nuevo Progreso: A. Mendoza R. et al., 2135 (UAMIZ).

Cyatheaceae

$*{ }^{\text {ta }}$ Cyathea costaricensis (Mett. ex Kuhn) Domin.

San Juan Colorado: A. Mendoza R. et al., 1854 (UAMIZ); Nuevo Progreso: A. Mendoza R. et al., 1865 (UAMIZ), 2115 (UAMIZ).

*ta Cyathea myosuroides (Liebm.) Domin.

Nuevo Progreso: A. Mendoza R. et al., 2141 (UAMIZ).

$*+{ }^{\text {ta }}$ Sphaeropteris horrida (Liebm.) R. M Tryon

Nuevo Progreso: A. Mendoza R. et al., 2137 (UAMIZ).

Dennstaedtiaceae

*t Pteridium aquilinum (L.) Kuhn var. feei (W. Schaffn. ex Fée) Maxon Nuevo Progreso: A. Mendoza R. et al., 2130 (UAMIZ).

Dryopteridaceae

* Bolbitis portoricensis (Spreng.) Hennipman

Nuevo Progreso: A. Mendoza R. et al., 1873 (UAMIZ), 2109

(UAMIZ); PeÑas Negras: A. Mendoza R. et al., 1886 (UAMIZ), 2013

(UAMIZ).

*t Ctenitis excelsa (Desv.) Proctor

Agua fría:A. Mendoza R. et al., 2042 (UAMIZ); Nuevo Progreso: A. Mendoza R. et al., 1872 (UAMIZ), 1877 (UAMIZ), 2133 (UAMIZ);

Peñas Negras: A. Mendoza R. et al., 2014 (UAMIZ).

${ }^{* t}$ Ctenitis interjecta (C. Chr.) Ching

Santa María Nutio: I. Mejía M. et al., 96 (UAMIZ).

*t Dryopteris karwinskyana (Mett.) Kuntze

PeÑas Negras: A. Mendoza R. et al., 2008 (UAMIZ).

*t Elaphoglossum sartorii (Liebm.) Mickel

Nuevo Progreso: A. Mendoza R. et al., 2117 (UAMIZ), 2134

(UAMIZ).

Gleicheniaceae

* Dicranopteris flexuosa (Schrad.) Underw.

Nuevo Progreso: A. Mendoza R. et al., 2121 (UAMIZ).

*t Gleichenella pectinata (Willd.) Ching

Nuevo Progreso: A. Mendoza R. et al., 1874 (UAMIZ), 2123

(UAMIZ).

*t Sticherus bifidus (Willd.) Ching

Nuevo Progreso: A. Mendoza R. et al., 2122 (UAMIZ).

Lygodiaceae

${ }^{\mathrm{t}}$ Lygodium venustum $\mathrm{Sw}$.

Nuevo Progreso: A. Mendoza R. et al., 1870 (UAMIZ), 2111

(UAMIZ); PeÑas Negras: A. Mendoza R. et al., 1882 (UAMIZ), 2012

(UAMIZ), 2151 (UAMIZ); SAN JUAN COLORADO: A. Mendoza R. et al., 1855 (UAMIZ). 
Polypodiaceae

*e Phlebodium decumanum (Willd.) J. Sm.

Nuevo Progreso: A. Mendoza R. et al. s.n. (UAMIZ).

*e Phlebodium inaequale T. Moore*

(=Polypodium pleurosorum Kunze ex Mett.)

Agua Fría: A. Mendoza R. et al., 2047, 2052 (UAMIZ); PeÑas

Negras: A. Mendoza R. et al., 2027 (UAMIZ).

*e Pleopeltis astrolepis (Liebm.) E. Fourn.

Nuevo Progreso: A. Mendoza R. et al., 2131 (UAMIZ).

*e,r Pleopeltis furfuracea (Schltdl. \& Cham.) A. R. Sm. \& Tejero (=Polypodium furfuraceum Schltdl. \& Cham.)

Agua Fría: A. Mendoza R. et al., 2051 (UAMIZ); PeÑas Negras: A.

Mendoza R. et al., 1885 (UAMIZ), 2029 (UAMIZ), 2149 (UAMIZ).

*e,r Polypodium colpodes Kunze

Agua Fría: A. Mendoza R. et al., 2050 (UAMIZ); PeÑas Negras: A.

Mendoza R. et al., 2018 (UAMIZ), 2027-BIS (UAMIZ).

${ }^{*}$ Polypodium plesiosorum Kunze

Peñas Negras: A. Mendoza R. et al., 2026 (UAMIZ).

Pteridaceae

*t Adiantopsis radiata $(\mathrm{L}$.) Fée

Nuevo Progreso: A. Mendoza R. et al., 1868 (UAMIZ), 2113

(UAMIZ), 2119 (UAMIZ).

*t Adiantum amplum $\mathrm{C}$. Presl

Agua Fría: A. Mendoza R. et al., 2038 (UAMIZ); Nuevo Progreso: A. Mendoza R. et al., 2145 (UAMIZ); Pena Negra: A. Mendoza R. et al., 2017 (UAMIZ), 2024 (UAMIZ); SAN JUAN COLORADO: A. Mendoza R. et al., 1864 (UAMIZ).

'Adiantum concinnum Humb. \& Bonpl. ex Willd.

Agua Fría: A. Mendoza R. et al., 2060 (UAMIZ); Nuevo Progreso: A. Mendoza R. et al., 2136 (UAMIZ); Peñas Negras: A. Mendoza R. et al., 2011 (UAMIZ).

**t Adiantum decoratum Maxon \& Weath.

SAN JuAn Colorado: A. Mendoza R. et al., 1862 (UAMIZ).

*tAdiantum galeottianum Hook.

Agua Fría: A. Mendoza R. et al., 2035-BIS (UAMIZ); SAN JUAN

Colorado: A. Mendoza R. et al., 2035 (UAMIZ).

${ }^{\mathrm{t}}$ Adiantum latifolium Lam.

Nuevo Progreso: A. Mendoza R. et al., 2118 (UAMIZ); SAn Juan

Colorado: A. Mendoza R. et al., 1860 (UAMIZ).

'Adiantum lunulatum Burm. $\mathrm{f}$.

Agua Fría: A. Mendoza R. et al., 2037 (UAMIZ); Peñas Negras: A.

Mendoza R. et al., 2016 (UAMIZ).

* $\mathrm{t}$ Adiantum patens Willd.

Agua Fría:A. Mendoza R. et al., 2044 (UAMIZ)

Nuevo Progreso: A. Mendoza R. et al., 2146 (UAMIZ); Peñas

Negras: A. Mendoza R. et al., 2028 (UAMIZ).

*tAdiantum petiolatum Desv.

SAn Juan Colorado: A. Mendoza R. et al., 1861 (UAMIZ).

* Adiantum trapeziforme $\mathrm{L}$.

Agua Fría: A. Mendoza R. et al., 2049 (UAMIZ); Nuevo Progreso:

A. Mendoza R. et al., 1869 (UAMIZ); Peñas Negras: A. Mendoza R. et al., 1884 (UAMIZ), 2150 (UAMIZ).

*e,r Ananthacorus angustifolius (Sw.) Underw. \& Maxon

Agua Fría: A. Mendoza R. et al., 2048 (UAMIZ), 2055 (UAMIZ);

Nuevo Progreso: A. Mendoza R. et al., 1876 (UAMIZ), 2114

(UAMIZ); SAN JUAN Colorado: I. Mejía M. et al., 113 (UAMIZ).

${ }^{\mathrm{t} C h e i l a n t h e s ~ l o z a n o i ~(M a x o n) ~ R . ~ M . ~ T r y o n ~ \& ~ A . ~}$

F. Tryon var. lozanoi

Peñas Negras: A. Mendoza R. et al., 2021 (UAMIZ).

${ }^{\mathrm{t}}$ Gaga angustifolia (Kunth) Fay-Wei Li \& Windham

(=Cheilanthes angustifolia Kunth)

San Juan Colorado: A. Mendoza R. 2033 (UAMIZ); Nuevo

Progreso: A. Mendoza R. et al., 2139 (UAMIZ).

${ }^{\mathrm{t}}$ Gaga decomposita (M. Martens \& Galeotti) Fay-Wei Li \& Windham (=Cheilanthes decomposita (M. Martens \& Galeotti) Fée)

SAN JuAn COLORAdo: A. Mendoza R. 2031 (UAMIZ).

$* * \mathrm{t} \times$ Hemionanthes gryphus (Mickel) Mickel
AgUa Fría: A. Mendoza R. et al., 2039 (UAMIZ).

${ }^{\mathrm{t}}$ Hemionitis subcordata (D. C. Eaton ex Davenp.) Mickel

Agua Fría: A. Mendoza R. et al., 2057BIS (UAMIZ); PeÑas Negras:

A. Mendoza R. et al., 2009 (UAMIZ).

${ }^{\mathrm{t}}$ Pityrogramma calomelanos (L.) Link

Agua Fría: A. Mendoza R. et al., 2053 (UAMIZ); Nuevo Progreso:

A. Mendoza R. et al., 2127 (UAMIZ); SAn JuAn Colorado: I. Mejía

M. et al., 115 (UAMIZ).

* Pteris biaurita $\mathrm{L}$.

Nuevo Progreso: A. Mendoza R. et al., 1871 (UAMIZ), 2112

(UAMIZ).

*t Pteris erosa Mickel \& Beitel

Nuevo Progreso: A. Mendoza R. et al., 1875 (UAMIZ).

*e Vittaria gramminifolia Kaulf.

Nuevo Progreso: A. Mendoza R. et al., 2147 (UAMIZ).

Tectariaceae

*t Tectaria incisa Cav. f. incisa

SAn Juan Colorado: A. Mendoza R. et al., 1853 (UAMIZ).

*t Tectaria mexicana (Fée) C. V. Morton

Agua Fría: I. Mejía M. et al., 127 (UAMIZ); A. Mendoza R. et al., 2043

(UAMIZ); Nuevo Progreso: A. Mendoza R. et al., 1881 (UAMIZ);

Peñas Negras: A. Mendoza R. et al., 1883 (UAMIZ), 2023 (UAMIZ).

Thelypteridaceae

*t Macrothelypteris torresiana (Gaudich.) Ching

Nuevo Progreso: A. Mendoza R. et al., 2128 (UAMIZ); SAn Juan

Colorado: A. Mendoza R. et al., 1863 (UAMIZ).

*t Thelypteris dentata (Forssk.) E. P. St. John

SAN JuAn Colorado: A. Mendoza R. et al., 1851BIS (UAMIZ).

*t Thelypteris hispidula (Decne.) C. F. Reed

Peñas Negras:A. Mendoza R. et al.1888 (UAMIZ), 1889 (UAMIZ);

SAN JuAn Colorado: A. Mendoza R. et al., 1852 (UAMIZ), 1858

(UAMIZ).

*t Thelypteris obliterata (Sw.) Proctor

Agua Fría: A. Mendoza R. et al., 2041 (UAMIZ); Nuevo Progreso:

A. Mendoza R. et al., 2110 (UAMIZ), 2129 (UAMIZ).

*t Thelypteris oligocarpa (Humb. \& Bonpl. ex Willd.) Ching

Nuevo Progreso: A. Mendoza R. et al., 2140 (UAMIZ).

*t Thelypteris patens $(\mathrm{Sw}$.) Small

Nuevo Progreso: A. Mendoza R. et al., 2144 (UAMIZ).

*t Thelypteris resinifera (Desv.) Proctor var. resinifera

Agua Fría:A. Mendoza R. et al., 2054 (UAMIZ); Nuevo Progreso: A.

Mendoza R. et al., 2132 (UAMIZ); PeÑas Negras: A. Mendoza R.

et al., 1890 (UAMIZ); SAn JuAn Colorado: A. Mendoza R. et al.,

1856 (UAMIZ), 1867 (UAMIZ).

*s Thelypteris serrata (Cav.) Alston

Nuevo Progreso: A. Mendoza R. et al., 1878 (UAMIZ); Peñas

Negras: A. Mendoza R. et al., 2030 (UAMIZ).

${ }^{\mathrm{t}}$ Thelypteris tetragona $(\mathrm{Sw}$.$) Small$

Nuevo Progreso: A. Mendoza R. et al., 1879 (UAMIZ); San Juan

Colorado: A. Mendoza R. et al., 1851, 1859 (UAMIZ).

\section{Referencias}

Acebey, A. R., Krömer, T., Vázquez-Torres, M. y Tejero-Díez, J. D. (2015). Helechos y licófitos de la Reserva de la Biosfera Los Tuxtlas, Veracruz, México. Botanical Sciences, 93, 313-344.

Acosta, S., Flores, A., Saynes, A., Aguilar, R. y Manzanero, G. (2003). Vegetación y flora de una zona semiárida de la cuenca alta del río Tehuantepec, Oaxaca, México. Polibotánica, 16, 125-152.

Aparicio-Lechuga, M. y Vargas-Fuerte V. (2010). Estudio florístico y uso de plantas vasculares en los terrenos comunales de Ciudad Ixtepec, Oaxaca. Tesis. Universidad Autónoma Chapingo, Estado de México.

Carrillo-Hernández, E. (2013). Listado florístico de la porción noroeste del distrito mixe de Oaxaca. Tesis. Universidad Autónoma Chapingo, Estado de México. 
Carvajal-Hernández, C., Krömer, T. y Vázquez-Torres, M. (2015). Riqueza y distribución de helechos y licófitos en el gradiente altitudinal del Cofre de Perote, centro de Veracruz, México. Botanical Sciences, 93, 601-614.

Plan Municipal de Desarrollo, San Juan Colorado, Oaxaca 20112013. San Juan Colorado [consultado 5 Jun 2015]. Disponible en: https://finanzasoaxaca.gob.mx/pdf/inversion_publica/pmds/11_13/188.pdf

Figueroa-Brito, S. y Guzmán-Rivera, F.Y. (2005). Listado florístico en el municipio de Capulalpam de Méndez, Distrito de Ixtlán de Juárez, Oaxaca. Tesis. Facultad de Estudios Superiores Los Reyes Iztacala, Universidad Nacional Autónoma de México, Estado de México.

García-Mendoza, A. J. (2004). Integración del conocimiento florístico del estado. En A. J. García-Mendoza, M. J. Ordóñez, y M. Briones-Salas (Eds.), Biodiversidad de Oaxaca (pp. 305-325). México, D.F.: Instituto de Biología, UNAM-Fondo Oaxaqueño para la Conservación de la Naturaleza, World Wildlife Fund.

García-Mendoza, A. J., y Meave, J. A. (Eds.). (2011). Diversidad florística de Oaxaca: de musgos a angiospermas (colecciones y lista de especies). México, D.F.: Universidad Nacional Autónoma de México-Comisión Nacional para el Conocimiento y Uso de la Biodiversidad.

Guízar-Nolazco, E. (2011). La vegetación de la Mixteca. Tesis doctoral. Universidad Autónoma Metropolitana-Iztapalapa, México, D.F.

Inegi (Instituto Nacional de Estadística, Geografía e Informática). (2005). Marco geoestadístico municipal. Prontuario de información geográfica de los Estados Unidos Mexicanos, San Juan Colorado, Oaxaca. Clave geoestadística 20188. Aguascalientes: Instituto Nacional de Estadística, Geografía y Estadística.

Inegi (Instituto Nacional de Estadística, Geografía e Informática). (2010). Compendio de información geográfica municipal 2010. San Juan Colorado, Oaxaca. Aguascalientes: Instituto Nacional de Estadística, Geografía e Informática [consultado 10 Jul 2015]. Disponible en: http://www.inegi.org.mx/geo/contenidos/topografia/compendio.aspx

Lorea-Hernández, F. y Munn-Estrada, D. X. (2005). Estudio florístico de los bosques mesófilos de la sierra Mazateca de Oaxaca, México. México, D.F. Instituto de Ecología, A.C., Comisión Nacional para el Conocimiento y Uso de la Biodiversidad.

Mejía-Marín, M.A. (2014). Riqueza y distribución de las monocotiledóneas nativas de San Juan Colorado, Oaxaca, México. Tesis de Maestría. Universidad Autónoma Metropolitana-Iztapalapa, México, DF.

Mickel, J. T. y Beitel, J. M. (1988). Pteridophyte flora of Oaxaca, Mexico. Memoirs of the New York Botanical Garden, 46, 1-568.

Mickel, J. T. y Smith, A. R. (2004). Pteridophyte flora of Oaxaca, Mexico. Memoirs of the New York Botanical Garden, 88, 1-1054.

Øllgaard, B. (2012). New combinations in Neotropical Lycopodiaceae. Phytotaxa, 57, 10-22.

Pérez-García, E. A., Meave, J. A. y Gallardo, C. (2001). Vegetación y flora de la región de Nizanda, Istmo de Tehuantepec, Oaxaca, México. Acta Botánica Mexicana, 56, 19-88.

Reyes-Santiago, J.P. (1993). Estudio florístico y fitogeográfico en el municipio de San Juan Mixtepec, Distrito Juxtlahuaca, Oaxaca. Tesis. Facultad de Estudios Superiores Los Reyes Iztacala, Universidad Nacional Autónoma de México, Estado de México.

Riba, R. y Lira, R. (1996). Pteridophyta. Flora del Valle de Tehuacán-Cuicatlán: fascículo 10. México, D.F.: Instituto de Biología, Universidad Nacional Autónoma de México.

Röpert, D. (ed.). (2000). [actualizado continuamente]. Digital specimen imagen at the herbarium Berolinense [consultado 5 Jun 2015]. Disponible en: http://ww2.bgbm.org/herbarium/default.cfm

Rzedowski, J. (2006). Vegetación de México, edición digital. Ciudad de México. Comisión Nacional para el Conocimiento y Uso de la Biodiversidad.
Salas-Morales, S. H., Saynes-Vásquez, A. y Schibli, L. (2003). Flora de la costa de Oaxaca: lista florística de la región de Zimatán. Boletín de la Sociedad Botánica de México, 72, 21-58.

Salas-Morales, S. H., Schibli, L., Nava-Zafra, A. y Saynes-Vásquez, A. (2007). Flora de la costa de Oaxaca, México (2): lista florística comentada del Parque Nacional Huatulco. Boletín de la Sociedad Botánica de México, 81, $101-130$.

Santiago-Cruz, M. (2013). La pteridoflora de la comunidad de Santa Cruz Yagavila, Municipio de Ixtlán de Juárez, Oaxaca. Tesis. Universidad de la Sierra de Juárez.

Semarnat, 2010. Norma Oficial Mexicana NOM-059-SEMARNAT-2010, Protección ambiental - Especies nativas de México de flora y fauna silvestres Categorías de riesgo y especificaciones para su inclusión, exclusión o cambio - Lista de especies en riesgo. Diario Oficial de la Federación. 30 de diciembre de 2010, Segunda Sección, México.

Smith, A. R., Pryer, K. M., Schuettpelz, E., Korall, P., Schneider, H. y Wolf, P. G. (2006). A classification for extant ferns. Taxon, 55, 705-731.

Smith, A. R. y Tejero-Díez, J. D. (2014). Pleopeltis (Polypodiaceae), a redefinition of the genus and nomenclatural novelties. Botanical Sciences, 92, $43-58$.

Solano-Hernández, L. (1997). Estudio florístico y descripción de la vegetación del municipio de Asunción Cuyotepeji, Distrito de Huajuapan de León, Oaxaca. México. Polibotánica, 5, 37-75.

Tejero-Díez, J. D. y Mickel, J. T. (2004). Las Pteridofitas. En A. J. GarcíaMendoza, M. J. Ordóñez-Díaz, y M. A. Briones-Salas (Eds.), Biodiversidad de Oaxaca (pp. 121-139). México, D.F.: Instituto de Biología, UNAM, Fondo Oaxaqueño para la conservación de la naturaleza y World Wildlife Found.

Tejero-Díez, J. D. y Mickel, J. T. (2011a). Lycopodiophyta. En A. J. GarcíaMendoza y J. A. Meave (Eds.), Diversidad florística de Oaxaca: de musgos a angiospermas (colecciones y listas de especies) (pp. 149-150). México, D.F.: Instituto de Biología, Universidad Nacional Autónoma de México/Comisión Nacional para el Conocimiento y Uso de la Biodiversidad.

Tejero-Díez, J. D. y Mickel, J. T. (2011b). Polypodiophyta. En A. J. GarcíaMendoza y J. A. Meave (Eds.), Diversidad florística de Oaxaca: de musgos a angiospermas (colecciones y listas de especies) (pp. 151-164). México, D.F.: Instituto de Biología, Universidad Nacional Autónoma de México/Comisión Nacional para el Conocimiento y Uso de la Biodiversidad.

The International Plant Names Index. (2015) [consultado Jun 2015]. Disponible en: http://www.ipni.org

The New York Botanical Garden. Catalogs specimens of vascular plants. (2015) [consultado Jun 2015]. Disponible en: http://sciweb.nybg.org/science2/ hcol/allvasc/index.asp.html

The Plant List (2013). Version 1.1 [consultado Jun 2015]. Disponible en: http://www.theplantlist.org

Thiers, B. [actualizado continuamente]. Index Herbariorum: a global directory of public herbaria and associated staff. New York Botanical Garden's Virtual Herbarium [consultado Jun 2015]. Disponible en: http://sweetgum.nybg.org/science/ih/

Torres-Colín, R., Torres-Colín, L., Dávila-Aranda, P. y Villaseñor-Ríos, J. L. (1997). Listados florísticos de México XVI. Flora del Distrito de Tehuantepec, Oaxaca. México, D.F.: Instituto de Biología, Universidad Nacional Autónoma de México.

Velázquez-Montes, E. (2009). Pteridophyta II. Flora del Valle de TehuacánCuicatlán. Fascículo 67. México, D.F.: Instituto de Biología, Universidad Nacional Autónoma de México.

Velázquez-Montes, E. (2010). Pteridaceae. Flora del Valle de TehuacánCuicatlán. Fascículo 80. México, D.F.: Instituto de Biología, Universidad Nacional Autónoma de México. 